\title{
Prevalencia de ictericia neonatal y factores asociados en recién nacidos a término.
}

\author{
Prevalence of Neonatal Jaundice and risk factors associated in \\ full-term newborns.
}

Ñacari Vera Miguel ${ }^{\text {1,a,b }}$

1. Universidad Nacional San Luis Gonzaga de Ica, Perú

a. Médico Residente Pediatría

b. Médico Cirujano

\section{Correspondencia:}

Miguel Giovani Ñacari Vera. Número de celular: 949146111 Correo Electrónico:

miguelnacari@hotmail.com Domicilio: Urb San Joaquin Mza B lote 28 - Ica,Perú.

\section{Contribuciones De Autoría:}

MÑV: Contribuciones en la concepción y diseño del manuscrito, recolección, análisis e interpretación de los datos, redacción y revisión crítica del contenido del manuscrito y aprobación final del artículo.

Conflicto De Intereses: No declarados.

Financiamiento: Autofinanciado.

\section{Como Citar}

Nacari-Vera Miguel. Prevalencia de ictericia neonatal y factores asociados en recién nacidos a término. Rev méd panacea 2018; (2): 63-68

Recibido: 26 - 04 - 2018

Aceptado: 30 - 05 - 2018

Publicado: 25 - 08 - 2018

\section{RESUMEN}

Objetivo: Describir la prevalencia y factores asociados a la ictericia neonatal en recién nacidos. Materiales y métodos: Estudio de revisión, descriptivo transversal. Las bases de datos de revistas médicas científicas empleadas principalmente fueron PubMed y Scielo. Resultados: Hasta la actualidad a nivel mundial se reportan casos de hiperbilirrubinemia neonatal. Las últimas prevalencias encontradas en la literatura son 55,2\% en Estados Unidos, 29 \% en Nepal, 6,7\% en Nigeria y en Europa valores que van del $6 \%$ al $59 \%$. Mientras que en América del Sur Bolivia y Chile han reportado altas prevalencias de $76,3 \%$ y $69,2 \%$, y en Perú alrededor de $7 \%$. Entre los factores de riesgo asociados principalmente se detallan bebé de sexo masculino, grupo sanguíneo del sistema $A B O$ o de factor Rh, defectos de la membrana del eritrocito (esferocitosis hereditaria), deficiencias enzimáticas (deficiencia de glucosa 6 fosfato deshidrogenasa) y hemoglobinopatías. Conclusiones: La prevalencia de ictericia neonatal es variable a nivel mundial. Los pocos estudios realizados en Perú reportan prevalencia alrededor del $7 \%$. Es necesario que se realicen más estudios para tener datos a nivel nacional que permitan establecer estrategias de control y tratamiento según las necesidades de la región.

\section{Palabras clave: Hiperbilirrubinemia neonatal, prevalencia, factores de riesgo}

\section{ABSTRACT}

Objective: To describe the prevalence and problems associated with neonatal jaundice. Materials and methods: Review study, cross-sectional descriptive. PubMed and Scielo were used as data bases. Results: 5 Up to now, cases of neonatal hyperbilirubinemia are reported worldwide. The last prevalences found in the literature were 55.2 in the United States, 29\% in Nepal, 6.7 in Nigeria, in Europe values ranging from $6 \%$ to $59 \%$. While in South America Bolivia and Chile have reported high prevalences of $76.3 \%$ and $69.2 \%$, and in Peru around $7 \%$. Among the associated risk factors are mainly male baby, blood group of the $A B O$ system or of the Rh factor, defects of the erythrocyte membrane (hereditary spherocytosis), enzymatic deficiencies (deficiency of glucose 6 phosphate dehydrogenase) and hemoglobinopathies

Conclusions: The prevalence of neonatal jaundice is variable worldwide. The few studies conducted in Peru report a prevalence around $7 \%$. It is necessary to carry out more studies in order to have data at the national level that allow establishing control and treatment strategies according to the needs of the region.

Keywords: Neonatal jaundice, prevalence, risk factors. 


\section{INTRODUCCIÓN}

La ictericia neonatal se caracteriza por una elevación de los niveles de bilirrubina sérica en los recién nacidos, produciéndose una acumulación del pigmento amarillo-naranja de la bilirrubina en la piel, las escleróticas, y otros tejidos, sin que ello implique ninguna causalidad en particular. $(1,2)$

La hiperbilirrubinemia neonatal se debe principalmente a la acumulación de bilirrubina, como resultado de alteraciones metabólicas causadas por diversos factores. Los recién nacidos, especialmente los pretérminos, tienen una conjugación y excreción de bilirrubina a través del hígado inmaduras. Normalmente, los niveles de bilirrubina sérica aumentan entre el tercer y quinto día de vida, y luego comenzarán a disminuir. $(2,3)$

La ictericia en los neonatos a término y más aún en los neonatos pretérmino muestra características clínicas que hay que considerar, así, depende de su temprano reconocimiento y la instauración de una terapéutica adecuada, las cuales son tareas habituales para el neonatólogo y el pediatra. Los procedimientos diagnósticos tienden a diferenciar las Ictericias Fisiológicas de las Ictericias no Fisiológicas. $(4,5)$

Existe controversia en relación a las posibles consecuencias de la ictericia neonatal y sobre el momento en que se debe iniciar su tratamiento, el dilema está en definir los niveles de bilirrubina para intervenir, la decisión está influenciada por la edad gestacional del niño, presencia de patologías o factores hematológicos predisponentes. En todo caso, la toma de decisiones en la conducción de un paciente neonatal con ictericia será más acertadas si cada situación se considerase individualmente, valorando en profundidad toda la información disponible y canalizando el cuadro clínico adecuadamente. $(2,6)$

Debido a su frecuencia y por el impacto que puede causar a nivel poblacional y a nivel cognitivo y por las complicaciones potenciales que podrían aparecer si no se conoce acerca de esta entidad clínica y debido a la poca información a nivel regional con la que se cuenta, se decidió investigar esta patología en nuestro medio.

\section{MATERIALES Y MÉTODOS}

La presente revisión representa un estudio descriptivo y transversal en el cual se detalla literatura relevante concerniente a la prevalencia de la ictericia neonatal y los factores asociados descritos hasta la actualidad. Se empleó el navegador Chrome y la búsqueda fue realizado usando base de datos que cuenten con revistas científicas indizadas tales como PubMed y Scielo principalmente.

Otros criterios de búsqueda y selección de artículos científicos incluyeron la fecha de publicación (dentro de los últimos cinco años), el empleo de los términos "ictericia neonatal", " prevalencia de ictericia neonatal y factores de riesgo" y "neonatal jaundice" principalmente.

\section{DESARROLLO Y DISCUSION}

Definición: La ictericia neonatal es la coloración amarillenta de la piel y/o escleras en los recién nacidos causada por el depósito de bilirrubina en los tejidos y es una de las causas más frecuentes de internamientos de bebés después del nacimiento (7). La bilirrubina es producto del catabolismo del grupo heme. Aproximadamente el 80 a 90 por ciento de la bilirrubina es producida durante la destrucción de la hemoglobina procedente de células de la serie roja viejas o de la eritropoyesis ineficaz. El otro 10 a 20 por ciento proviene de otras proteínas que contienen al grupo heme como los citocromos, las catalasas, entre otras. $(8,9)$. Cuando ocurren variaciones en el metabolismo de la bilirrubina se produce la ictericia neonatal, $y$, dependiendo de estas variaciones la ictericia puede ser fisiológica o patológica (10). Altos niveles de bilirrubina acumulada en la piel y en la sangre del bebé producen la ictericia neonatal y según el CDC el $60 \%$ de los bebés a término y el $80 \%$ de los bebés pre término presentarán esta condición (11).

Tipos de ictericia neonatal: La literatura describe dos tipos de ictericia: la fisiológica y la patológica. La ictericia fisiológica es aquella relacionada a hiperbilirrubinemia no conjugada leve y afecta casi a un gran porcentaje de recién nacidos y que se resuelve en pocas semanas luego del nacimiento. Esta condición es causada por aumento en la producción de la bilirrubina, disminución en su excreción e incremento de su circulación enterohepática. El pico de bilirrubina sérica total en la ictericia fisiológica es típicamente 5 a $6 \mathrm{mg} / \mathrm{dl}$ (86 a 103 umol/L) ocurre entre las 72 y 96 horas de vida, y no debe exceder de 17 a 18 mg/dl (291 a $308 \mu \mathrm{mol} / \mathrm{L}$ ). Niveles mayores de bilirrubinemia no conjugada son patológicos y pueden ocurrir en varias condiciones anormales. $(12,8)$. La producción de bilirrubina en los recién nacidos es 2 a 3 veces mayor que en los adultos, debido al rápido recambio de células rojas. La excreción de bilirrubina está disminuida en los recién nacidos por deficiencia de la enzima glucoroniltransferasa (UGT) cuya actividad en los neonatos es aproximadamente el 1 por ciento de la de los adultos. (13). Los recién nacidos tienen menor colonización bacteriana intestinal que los adultos, lo que reduce su capacidad para reducir la bilirrubina a urobilinógeno generando altas concentraciones de bilirrubina en el intestino. Además, la actividad de la betaglucuronidasa está aumentada en los recién nacidos, aumentando la hidrólisis de la bilirrubina conjugada y su conversión a no conjugada. La bilirrubina no conjugada es reabsorbida en el proceso conocido como circulación enterohepática. (3). Otros factores como las variaciones genéticas específicas en la habilidad para la conjugación incrementan la circulación entero-hepática, y pueden contribuir a la severidad de la ictericia fisiológica en algunos recién nacidos. Variaciones o polimorfismos en el gen codificador de UGT (UGT1A1) contribuye a las diferencias raciales. Es causa de ictericia la mutación común del gen UGT 
(Gly71Arg) lo que ocurre en asiáticos e incrementa la incidencia de ictericia severa en más o menos un $20 \%$. (4). La mayoría de los casos de ictericia patológica son causados por una exageración de los mecanismos que producen la ictericia fisiológica, por tal motivo, es necesario diferenciarla. Los siguientes hallazgos sugieren ictericia patológica: Ictericia en las primeras $24 \mathrm{hrs}$ (usualmente causada por incremento en la producción), niveles de bilirrubina total (BT) por encima del 95 percentil para la edad, bilirrubina conjugada $>1.0 \mathrm{mg} / \mathrm{dL}$ $(17.1 \mu \mathrm{mol} / \mathrm{L})$ si la BT es $5.0 \mathrm{mg} / \mathrm{dL}$ (85.5 $\mu \mathrm{mol} / \mathrm{L})$ y velocidad de incremento de la BT mayor $0.2 \mathrm{mg} / \mathrm{dL}$ (3.4 $\mu \mathrm{mol} / \mathrm{L})$ por hora (14).

La causa más común de hiperbilirrubinemia indirecta patológica es el incremento en la producción $(14,15)$. Los trastornos hemolíticos resultan de desórdenes inmunológicos (como la incompatibilidad $A B O$ o Rh) o defectos inherentes a la membrana del glóbulo rojo (como la esferocitosis hereditaria), deficiencias enzimáticas (como la deficiencia de glucosa 6 fosfato deshidrogenasa [G6PD]), o hemoglobinopatías $(2,6,4)$.

Otras causas de producción incrementada incluyen policitemia, hemorragias cerradas (como cefalohematoma), y algunas infecciones. Infantes macrosómicos de madres diabéticas también tienen incremento en la producción de bilirrubina $(9,14)$.

Adicionalmente una significativa disminución en la excreción de bilirrubina por el hígado es causada primariamente por defectos inherentes a la enzima UGT en los que se produce disminución en la conjugación de la bilirrubina. Estos desordenes incluyen el Síndrome de Crigler-Najjar tipo I y II y el Síndrome de Gilbert. $(3,8,14)$.

Aproximación clínica al niño de término con ictericia (15), objetivo de las consideraciones siguientes es saber cuándo sospechar que una hiperbilirrubinemia puede tener una causa patológica. Frente a todo recién nacido a término con ictericia debe seguirse las siguientes pautas para descartar en primer lugar una posible causa anormal de hiperbilirrubinemia: 1. Evaluar antecedentes perinatales en los casos de ictericia es importante preguntar en forma dirigida por niños anteriores con enfermedad hemolítica, por incompatibilidad $\mathrm{RH}$, de grupo clásico o de subgrupo; niños anteriores con hiperbilirrubinemia que requirieron tratamiento; antecedentes étnicos y geográficos que orienten a algunas causas genéticas de hiperbilirrubinemia como la deficiencia de glucosa 6 fosfato dehidrogenasa, síndrome de Crigler-Najjar, Talasemia, Esferocitosis familiar; antecedente de parto traumático $(16,8,4)$.

2. Precisar el momento de aparición de la ictericia y velocidad de ascenso de la bilirrubina. Toda ictericia de aparición precoz, antes de las 24 horas, debe considerarse patológica hasta no probarse lo contrario. Mientras más precoz, es más urgente evaluar la ictericia y considerar una eventual intervención, de acuerdo a su etiología. La hiperbilirrubinemia severa temprana, generalmente se asocia a una producción incrementada por factores hemolíticos y presentes antes de las 72 hrs de vida. Los niveles séricos de bilirrubina se encuentran por encima del percentil 75 en nomograma horario y representan un alto riesgo para el recién nacido. La hiperbilirrubinemia severa tardía, generalmente asociada a una eliminación disminuida, puede o no asociarse a incremento de su producción y presente luego de las 72 hrs vida. Generalmente, se debe a problemas de lactancia o leche materna, estreñimiento, mayor circulación enterohepática, etc. El cribado de bilirrubina al alta, la reconoce y de acuerdo a sus percentiles en el nomograma horario, se instituye el manejo correspondiente. $(3,4)$.

Por otro lado, la ictericia prolongada e ictericia de aparición tardía, se trata de dos situaciones que plantean la posibilidad de una ictericia patológica. De especial importancia es descartar una ictericia colestásica, ésta es siempre patológica. Se diagnostica por el ascenso de la bilirrubina directa sobre $2 \mathrm{mg} / \mathrm{dl}$. Se requiere de una acuciosa investigación etiológica y tratamiento específico. En el caso de la atresia de vías biliares, el resultado del tratamiento quirúrgico depende de que este se efectúe antes de las 8 semanas de vida. Alrededor de un 20 a 30\% de los niños alimentados al pecho presentan ictericia al $10^{\circ}$ día de vida, por lo que este hecho no puede ser considerado anormal. Si en este momento o antes el recién nacido presenta coluria o acolia, se debe medir la bilirrubina directa. Igual conducta se debe tener si la ictericia dura más de 15 días $(2,14,4)$.

\section{Exámenes de laboratorio:}

Grupo RH y Coombs directo, son los exámenes de mayor rendimiento para diagnosticar una enfermedad hemolítica por isoinmunización $\mathrm{Rh}$ o sub grupo. El Coombs directo tiene menor sensibilidad y valor predictivo para el caso de la enfermedad hemolítica por incompatibilidad de grupo clásico $(2,15,8)$.

Bilirrubinemia total. La decisión de tomar un examen de bilirrubina depende del momento de aparición y de la evolución de esta. Siempre debe hacerse si la ictericia aparece en las primeras 24 hrs. La intensidad se apreciará por el progreso céfalocaudal de ésta. En general, en toda ictericia que alcance a muslos y piernas se debe efectuar una bilirrubina total. La experiencia clínica es muy importante en estos casos. Ante la duda es mejor pedir el examen $(14,4)$.

La bilirrubinemia total es, junto al grupo Rh y Coombs el examen más útil para la evaluación de una ictericia. Nos informa sobre el nivel, la evolución, y es indispensable para decidir la necesidad de tratamiento. Es fundamental estar realizando controles de calidad y las calibraciones recomendadas para el método que se use. Es necesario repetirlo en casos que haya discordancia con la clínica (3, 8,6).

Análisis de sangre. Hemoglobina, hematocrito, recuento de reticulocitos, características del frotis y Coombs indirecto son exámenes que pueden ayudar. 
Todos tienen poca sensibilidad y especificidad para hacer el diagnóstico de un síndrome hemolítico. No es útil hacerlos de rutina en niños con hiperbilirrubinemia, sino que dejarlos como una ayuda para hiperbilirrubinemias importantes en que se sospecha hemólisis, que no son detectadas por el Coombs directo. El caso más frecuente, es el de la enfermedad hemolítica por incompatibilidad de grupo clásico para la cual no hay ningún examen con buena sensibilidad $y$ especificidad para diagnosticarla. La presencia de anemia, la precocidad de la ictericia y la velocidad de ascenso de la bilirrubina es lo más importante para el diagnóstico y la decisión de tratamiento. El Coombs directo ayuda, pero es poco sensible y con limitado valor predictivo. El valor del Coombs indirecto positivo se considera de escasa utilidad para la toma de decisiones $(16,15,8)$.

Tratamiento, actualmente se dispone de tres métodos de tratamiento, descritos brevemente a continuación

c.1 Exanguinotransfusión, empleado para la excreción de la bilirrubina via mecánica.

c.2 Terapia farmacológica, con el objetivo de mejorar e incrementar las vías metabólicas de excreción de la bilirrubina.

c.3 Fototerapia, también tiene objetivo la excreción de la bilirrubina disminuyendo el trabajo a nivel del hígado permitiendo que la vía metabólica renal se encargue de la eliminación de la bilirrubina. Este mecanismo emplea la luz para la destrucción física de la bilirrubina transformándola en un producto más pequeños (lumirrubina) que puede ser excretado fácilmente a través de la orina $(16,8,17)$.

Esta decisión debe considerar además de las condiciones clínicas del recién nacido, su situación social, geográfica y posibilidades de seguimiento $(8,16)$. La indicación de fototerapia o de seguimiento al alta con bilirrubinas la hará el médico de acuerdo a la prudente consideración de estos factores $(17,18)$.

\section{Epidemiología}

Diversas investigaciones se han publicado sobre la ictericia neonatal, un reciente estudio publicado en Estados Unidos en el año 2018, encontró que la prevalencia de ictericia neonatal fue de $55.2 \%$. Se reportó que hubo un $10 \%$ de bebés de raza negra, siendo diagnosticados con ictericia, no presentaron ictericia clínica. A nivel mundial se tiene reportado que el $10 \%$ de nacidos vivos requerirá fototerapia como tratamiento para la hiperbilirrubinemia neonatal, situación que se dificulta en zonas de bajos recursos económicos (como en el centro y sur de Asia) donde el acceso a un centro de salud, incluso en el momento del parto, es complicado o inexistente $(20,21)$. Bajo esta premisa se ha reportado que en Nepal hay una incidencia de ictericia neonatal de 29.3 por mil nacimientos vivos. Y dentro de los factores de riesgo con estadística significativa reportaron el peso al nacimiento, sexo del recién nacido, la dificultad de la alimentación, una prolongada labor de parto y ser primeriza, principalmente (21).
Una investigación descriptiva concluyó que en Nigeria fue el $6,7 \%$. En Europa, la hiperbilirrubinemia neonatal varía desde el $59 \%$ hallado en Suecia hasta el 28 '5\% en Roma, el $11 \%$ encontrado en Portugal, o el $6 \%$ de Grecia. Concluyendo que existe una elevada variabilidad debido al origen multicausal de la enfermedad. España presenta una baja incidencia de hiperbilirrubinemia neonatal, aunque la menor incidencia encontrada corresponde a Dinamarca. (22).

A nivel de América Latina, en México se ha reportado una prevalencia de hiperbilirrubinemia indirecta neonatal de $17 \%$ identificando como factores de riesgo asociados la edad gestacional menos de 35 semanas, la lactancia exclusiva y la sepsis (23). Mientras que entre los factores promotores de esta condición se menciona a la sepsis y el ayuno (24). Por otro lado, en La Habana, Cuba, encuentran como factores de riesgo principales a la prematuridad y el bajo peso al nacer. Estudios de prevalencia hiperbilirrubinemia neonatal en Bolivia y Chile mencionan valores similares, que van de $76.3 \%$ y $69,2 \%$ respectivamente, además hallaron que los nacidos de sexo masculino tuvieron una mayor frecuencia de esta condición, 61,9\% en Bolivia y 58,18\% en Chile de En Bolivia se presenta una prevalencia de $76.3 \%$, siendo más frecuente en bebés del sexo masculino (61.9\%).

Investigadores a nivel nacional han realizado investigaciones retrospectivas en Huancayo y Lima principalmente, hallando valores de prevalencia de 4,2\% en Huancayo y $6,03 \%$ en Lima $(26,30)$. Los casos de hiperbilirrubinemia neonatal fueron más frecuentes en bebés del sexo masculino, lo cual coincide con lo reportado en los países de Bolivia y Chile, como se mencionó en el párrafo anterior. $(26,27,28)$. La incidencia global de ictericia neonatal en el hospital San Bartolomé de Lima fue de 4.6\% en el año 2002 (30); sin embargo, esta no hace diferenciación entre el tiempo de aparición ni la intensidad. Los factores de riesgo que han sido relacionados a la aparición de ictericia temprana son incompatibilidad de grupo sanguíneo del sistema $A B O$ o de factor $\mathrm{Rh}$, defectos de la membrana del eritrocito (esferocitosis hereditaria), deficiencias enzimáticas (deficiencia de glucosa 6 fosfato deshidrogenasa) y hemoglobinopatías (29).

\section{CONCLUSIONES}

La casuística de hiperbilurrinemia neonatal es frecuente a nivel mundial reportándose diversos valores de prevalencia. En nuestro país, las pocas investigaciones realizadas en dos ciudades grandes como Huancayo y Lima muestran una prevalencia baja, sin embargo, es necesario realizar estudios que nos permiten recopilar mayor información en diferentes regiones de nuestro país ya que los principales factores de riesgo podrían ser otros en zonas más alejadas, de mayor pobreza y con limitados accesos a los recursos hospitalarios. Con mayor investigación nacional sobre los casos de hiperbilirrubinemia neonatal y sus factores de riesgo, las autoridades pertinentes podrán diseñar estrategias que permitan el control y tratamiento de esta enfermedad. 


\section{REFERENCIAS BIBLIOGRÁFICAS}

1. Fernández Rodríguez $M$, Martín Muñoz $P$. La valoración clínica de la ictericia no es buen método para el cribado de hiperbilirrubinemia neonatal. EvidPediar. 2008; 4: 7

2. Cohen RS, Ronald JW, Stevenson DK. Understanding Neonatal Jaundice: A Perspective on Causation. PediatrNeonatol. 2010; 51(3):143-48

3. Omeñaca Teres, F- González Gallardo, M. Servicio de neonatología. Hospital universitario la paz. Madrid. https://www.pediatriaintegral.es/wp-content/uploads /2014/xviii06/03/367-374.pdf

4. Dr. Gonzales Jose, Dra Hernandez S, Dr. Ruiz Cr Jaime Guía de Practica clínica Detección Oportuna, Diagnóstico y Tratamiento de la Hiperbilirrubinemia en Niños mayores de 35 semanas de gestación hasta las 2 semanas de vida extrauterina, México, Secretaria de Salud, 2009. Disponible en: http. // www.cenetec.salud.gob.mx/interior/gpc .html.

5. Quesada Cali, D - Arbulú Maurtua, P. Frecuencia de hiperbilirrubinemia neonatal en el Hospital José Carrasco Artega de Julio 2010 a Junio 2011. Cuenca Ecuador.

6. Vásquez de Kartzow $\mathrm{R}$, Martínez Orozco MX, Acosta Argoti F, Velasco López D. Ictericia Neonatal. Guías de Práctica Clínica basada en la Evidencia. Postgraduate Medicine 2009, 38(5): 233-50

7. Maamouri G, Boskabadi H, Mafinejad S, Bozorgnia Y, khakshur A. Efficacy of Oral Zinc Sulfate Intake in Prevention of Neonatal. Jaundice Iranian Journal of neonatology. 2014;4(4):11-6

8. Chavez $M$, Hiperbilirrubinemia en recién nacidos, causas, diagnóstico y tratamiento: estudio retrospectivo en 155 casos atendidos en el Hospital General Cayetano Heredia (2002). Tesis Br. Med. TB_UPCH; 2004, 105420, CH31

9. Jaundice May 2010, Funded to produce guidelines for the NHS by NICE. Disponible en: www.rcog.org.u

10. Centro nacional de Excelencia Tecnológica en Salud. Guía de Práctica Clínica Detección Oportuna, Diagnóstico y Tratamiento de la Hiperbilirrubinemia en Niños Mayores de 35 Semanas de Gestación Hasta las 2 Semanas de Vida Extrauterina, México; Secretaría de Salud, 2010. ISBN 978-607-8270-85-9

11. CDC. Jaundice. Breastfeeding. 2018. https://cdc.gov/breastfeeding/breastfeeding-specialcircumstances/maternal-or-infant-illnesses/jaundice. html

12. Reina Abrigo Raúl. Prevalencia y factores de riesgo para ictericia neonatal en el servicio de pediatría del hospital vitarte de enero 2013 a diciembre 2013. Asociación Universidad Privada San Juan Bautista Facultad de Ciencias de la Salud Escuela de Medicina Humana. Lima Perú de 2014

13. Cajamarca Berrezueta, C - Rojas Quito, S. Frecuencia de hiperbilirrubinemia por incompatiblidad $A B O$ en recién nacidos. Hospital Vicente Corral Moscoso. Cuenca- Periodo 2014-2015
14. Gomella TL, Cunningham D. Manual de Neonatología. Ed. 3 Panamericana. 2008.

15. Galván G. Incompatibilidad $A B O$ en neonatos sometidos a exanguineotransfusión en el Hospital San Bartolomé (Enero a Diciembre 2004) T.E. Pediatría Facultad de Med. UNFV. 44 pp., tablas, gráficas.

16. Birembaum HJ. Prophylactic Phototherapy in $A B O$ incompatibility. J. Perinatol; 2005, 25(9): 590-594.

17. Bhutáni VK, Brown AK. System-based approach to management of neonatal jaundice and prevention of kernicterus. J Pediatr 2002; 140(4): 396-403

18. Campo González Ana, Alonso Uría Rosa María, Amador Morán Rafael, Ballesté López Irka. Comparación de dos métodos diagnósticos de ictericia neonatal. Rev Cubana Pediatr [revista en la Internet]. 2012 Mar [citado 2015 Sep 20]; 84(1): 67-72.

19. Brits $H$, Adendorff J, Huisamen D, Beukes D, Botha $K$, Herbst $H$, et al. The prevalence of neonatal jaundice and risk factors in healthy term neonates at National District Hospital in Bloemfontein. Afr J Prim Health Care Fam Med. 2018;10(1):e1-e6.

20. Bhutani VK. Editorial: building evidence to manage newborn jaundice worldwide. Indian J Pediatr. 2012 Feb;79(2):253-255

21. Scrafford CG, Mullany LC, Katz J, Khatry SK, LeClerq SC, Darmstadt GL, et al. Incidence of and risk factors for neonatal jaundice among newborns in southern Nepal. Trop Med Int Health. 2013;18(11):1317-28.

22. Castano P, Sanchez M. Hiperbilirrubinemia Neonatal revisión de la situación actual. Revista científica de enfermería. Argentina 2011. Pág. 1 - 11

23. Villalobos - Acazar G, Guzman-Barcena J, Gonzales Perez V, Rojas Hernandez A, factores promotores de la hiperbilirrubinemia neonatal no hemolítica en una unidad de cuidados intensivos del recién nacido; Rev Perinatol Reprod Hum 2009; 15(3): 181 - 187.

24. Gallegos Dávila JA, Rodriguez Balderrama I, Rogelio Rodríguez B, Abrego Moya V, Rodríguez Camelo G; Prevalencia y factores de riesgo para hiperbilirrubinemia indirecta neonatal en un hospital universitario; Medicina Universitaria 2009; 11(45): 226-230

25. Calvimontes, C; Pinto S.; Rodas M.;SUarez L.; Tapia M.2014. Prevalencia de ictericia neonatal (hiperbilirrubinemia) en el Hospital Universitario, gestión 2013. ECOFARM. Bolivia-Sucre. https://ecorfan.org/bolivia/handbooks/ciencias\%20 de\%20la\%20salud\%20l/Articulo\%206.pdf

26. De la Cruz C. Prevalencia y factores de riesgo de ictericia en el Hospital Huancayo. Tesis para obtener el título de Médico cirujano. Universidad Peruana los Andes de Huancayo. Perú 2015

27. Carrasco S. Prevalencia de Ictericia Neonatal, (Hiperbilirrubinemia intermedia) y factores asociados en Recién nacidos a término en el Hospital 
II Ramón Castilla - EsSalud durante el año 2014. Tesis para optar el grado de Médico Cirujano. Universidad Ricardo Palma. Perú 2016.

28. Gonzales - Tipiana I. Macrosomía Fetal, prevalencia, factores de riesgo asociados y complicaciones en el Hospital Regional de Ica, Perú. Tesis Med UNSLG; 2012
29. 29. Baltazar T, Prevalencia y causas de ictericia neonatal en el hospital Nacional Ramiro Priale. Priale Huancayo en periodo 2014

30. 30. Cabrera K. Factores perinatales asociados a la presencia de ictericia patológica neonatal Hospital Vitarte 2014. Tesis para optar el título de especialista en pediatría. Universidad San Martin de Porres. Perú 2014.

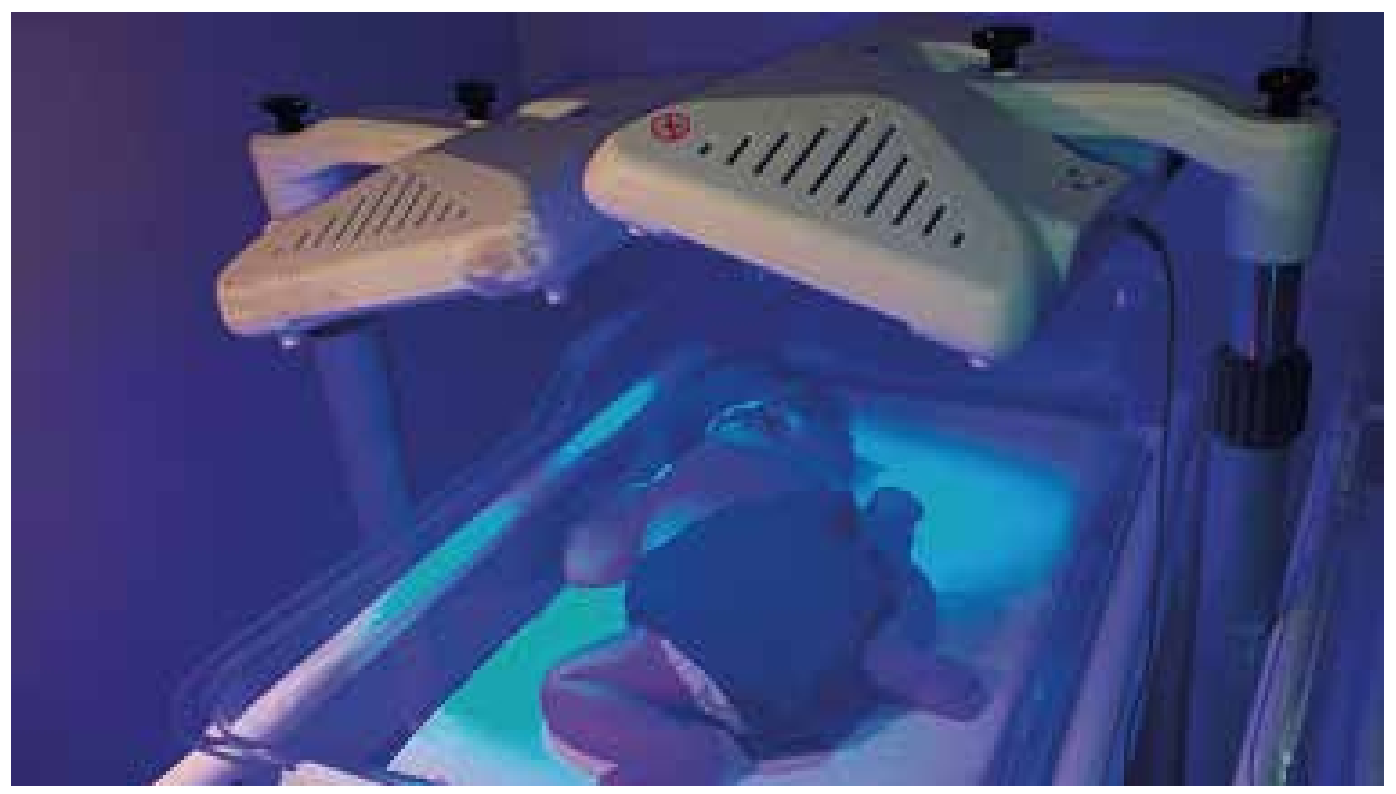

Fig 1. Recien nacido con ictericia en fototerapia. Hospital Santa Maria del Socorro. Ica

\section{Las ediciones anteriores de revista médica PANACEA están disponibles en:}

\section{www.revpanacea.unica.edu.pe}

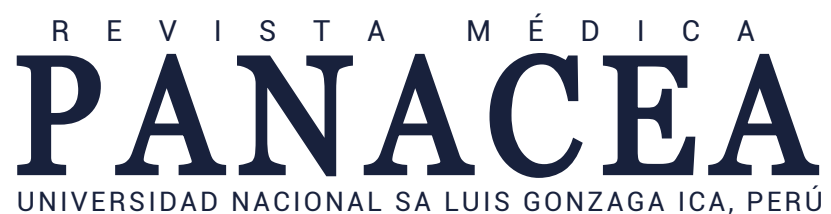

